\title{
Robust stop-and-go control strategy: an algebraic approach for nonlinear estimation and control
}

\section{Jorge VILLAGRA*}

Departamento de Ingenieria de Sistemas y Automatica, Universidad Carlos III, Leganes(Madrid), Spain

jvillagr@ing.uc3m.es

${ }^{*}$ Corresponding author

\section{Brigitte d'ANDRÉA-NOVEL Sungwoo CHOI}

Centre de Robotique, École des Mines de Paris

75272 Paris Cedex 06, France

brigitte.dandrea-novel@ensmp.fr

sung-woo.choi@ensmp.fr

\section{Michel FLIESS}

INRIA - ALIEN \& LIX (CNRS, UMR 7161), École polytechnique

91128 Palaiseau, France

Michel.Fliess@polytechnique.edu

\section{Hugues MOUNIER}

Institut d'Électronique Fondamentale (CNRS, UMR 8622)

Université Paris-Sud, 91405 Orsay, France

Hugues.Mounier@ief.u-psud.fr

\begin{abstract}
This paper describes a robust stop-and-go control strategy for vehicles. Since sensors used in a real automotive context are generally low cost, measurements are quite noisy. Furthermore, many vehicle/road interaction factors (road slope, rolling resistance, aerodynamic forces) are very poorly known. Hence, a robust strategy to noise and parameters is proposed within the same theoretical framework: algebraic nonlinear estimation and control techniques. On the one hand, noisy signals will be processed in order to obtain accurate derivatives, and thereafter, variable estimates. On the other hand, a grey-box closedloop control will be implemented to reject all kind of disturbances caused by exogenous parameter uncertainties.
\end{abstract}

Keywords: stop-and-go, robustness, nonlinear estimation, adaptive control, model-free control, PID controllers.

Biographical notes: Jorge Villagra received the M.Sc. degree from Universidad Politecnica de Madrid, Madrid, Spain, in 2002, and the $\mathrm{Ph} . \mathrm{D}$. degree in robotics, real-time computing, and automatic control from the École des Mines de Paris, Paris, France, in 2006. He is currently a Visiting Professor at the University Carlos III, Madrid, Spain. 
His research interests include nonlinear and optimal control in automotive applications and robust networked control systems in robotic applications.

Brigitte d'Andréa-Novel graduated from École Supérieure d'Informatique, Électronique, Automatique in 1984. She received the Ph. D. degree from École Nationale Supérieure des Mines de Paris in 1987 and the Habilitation degree from Université Paris-Sud in 1995. She is currently a Professor of Systems Control Theory and responsible for the research group in Advanced Control Systems at the Centre de Robotique - École des Mines de Paris. Her current research interests include nonlinear control theory and applications to underactuated mechanical systems, control of wheeled vehicles with applications to automated highways, and boundary control of dynamical systems coupling ODEs and PDEs.

Sungwoo Choi received the B.S. degree in electrical engineering from Korea Advanced Institute of Science and Technology (KAIST), Daejeon, Korea, in 2005 and the M.S. degree in Science and Executive Engineering from École Nationale Supérieure des Mines de Paris (MINES ParisTech) in 2007, Paris, France. He is currently a 3rd year Ph.D. candidate in Advanced Control System group at the Centre de Robotique, Mines ParisTech, and his research interests include vehicle dynamics and control, and driving assistance system.

Michel Fliess is a Research Director at the Centre National de la Recherche Scientifique and works at the École Polytechnique (Palaiseau, France). He is the head of the INRIA project called ALIEN, which is devoted to the study and the development of new techniques in identification and estimation. In 1991 he invented with J. Levine, P. Martin, and P. Rouchon, the notion of differentially flat systems which is playing a major role in control applications.

Hugues Mounier obtained his Ph.D. in automatic control in 1995 from the Laboratoire des Signaux et Systèmes of the Université Paris Sud, Orsay, France. From 1998 to now he has been with the Institut d'Electronique Fondamentale of the same University. He is currently the head of the AXIS department of this Institute. His research interests include automotive and real time control, delay systems and systems modelled by partial differential equations.

\section{Introduction}

\subsection{Generalities}

Car-following has become of increased importance in traffic engineering and safety research. Thus, adaptive cruise control (ACC) and stop-and-go control systems have been deeply studied in recent years [Vahidi and Eskandarian, 2003]. Let us recall that while ACC automatically accelerates or decelerates the vehicle to keep a quasi-constant target velocity and the headway distance, stop-and-go deals with the vehicle circulating in towns with frequent and sometimes hard stops and accelerations. It is not difficult to understand that both situations present completely different comfort and safety constraints, and therefore, that in most of the 
reported works, ACC and stop-and-go problems are treated separately.

Some approaches ([Persson et al, 1999], [Yi and Moon, 2004 ]) have tried to reproduce human behavior in order to achieve a 'comfort-based' control. Unfortunately, this kind of strategy may not necessarily lead to safe operation. Besides, external factors such as road characteristics, weather conditions, and traffic load should be taken into account in a robust and safe control system. On the other hand, an accepted comfort criteria is to guarantee bounded longitudinal accelerations and jerks.

Using this idea, many authors (cf. e.g. [Brackstone and McDonald, 2000], [Chien and Ioannou, 1992], [Hiraoka et al, 2005]) have modeled inter-distance using different types of time polynomials, whose coefficients are obtained respecting safety acceleration and jerk constraints.

In general, these approaches produce acceptable results in an ACC scenario. However, during a sudden deceleration of the preceding car, the vehicles present a large transitory relative velocity and the actual inter-distance decreases abruptly. Hence, this dynamical scenario would not be suitably represented by static polynomial models, but by some kind of inter-distance dynamic model.

[Martinez and Canudas-de-Wit, 2007] proposed a nonlinear reference model taking into account safe and comfort specification in an intuitive way. In addition, the model is combined with a simple feedback loop used to compensate unmodeled dynamics and external disturbances. However, this work makes two assumptions that are never met in real situations:

- the velocity and the acceleration of the leader vehicle are perfectly measured from suitable sensors;

- the reference acceleration generated by the dynamic inter-distance model is instantaneously applied to the following vehicle ${ }^{\mathrm{a}}$.

When measurements come uniquely from a radar, inter-distance and its time derivative measurement are specially corrupted. However, very few works consider this important issue.

Concerning the engine and break responses, it is clear that they cannot provide instantaneous actions fulfilling the desired acceleration. Hence, different approaches have been proposed to tackle the actuators nonlinear dynamics ${ }^{\mathrm{b}}$. Input/output linearization [Swaroop et al, 1994], fuzzy logic (cf. [Naranjo et al, 2006], [Germann and Isermann, 1995]) or sliding mode control (cf. [Gerdes and Hedrick, 1997] or [Nouvelière and Mammar, 2004]) have been used to deal with the engine control. Feedback linearization [Raza et al, 1997] and sliding modes [Yi and Chung, 2001] have also been implemented to control a nonlinear brake model.

Most of the above mentioned approaches are fixed gain controllers. However, vehicle parameters vary during the life time of the vehicle. Certain vehicle or road parameters, like rolling resistance, road inclination or friction coefficient, could change during a single trip. Examples of adaptive controller for vehicle control applications can be found in works by [Liubakka et al, 1993], [Ioannou and Xu, 1994], [Oda et al, 1991] or [Youcef-Toumi et al, 1992].

\footnotetext{
${ }^{a}$ Engine/brake torque generation from a reference acceleration is not an easy task in demanding situations.

${ }^{\mathrm{b}} \mathrm{cf}$. [Cho and Hedrick, 1995] and [Raza et al, 1997] for all details about engine and brake models, respectively.
} 


\subsection{Contributions}

The generation of the reference acceleration of the follower vehicle is obtained by the algorithm introduced in [Martinez and Canudas-de-Wit, 2007]. This will constitute a feedforward control in an ideal situation. Our contribution is twofold:

- We will elaborate realistic engine/brake torque to produce the expected reference acceleration of the follower vehicle

- Since measurement noises and unmodeled dynamics have to be taken into account, we will use a grey-box control strategy. Compared to classical PD controllers, as proposed in [Martinez and Canudas-de-Wit, 2007], the strength of this approach is that noise perturbations and neglected dynamics will be compensated with algebraic nonlinear estimation, which will lead to a straightforward tuning of the PD coefficients.

To compute the final closed-loop control, we need an estimation of unknown quantities, which is achieved via noise robust real time estimators, given by closedform algebraic formulae (cf. [Fliess et al, 2008]).

The proposed algebraic framework will be detailed in the appendix of the article, where an application to the vehicle velocities estimation will be also introduced.

\subsection{Outline of the paper}

The general control scheme will be presented in Section 2. In the third Section, the model-free control (i.e. the generalized grey-box closed-loop control) will be introduced. Section 4 will be devoted to recall vehicle dynamics and the feedforward control, where a longitudinal acceleration and a consequent torque is generated under ideal circumstances. This section also shows the motivation for the choice of algebraic techniques introduced in Section A. Noise and parameter robustness will be tackled with a grey-box closed-loop control approach in Section 4. Simulation results will show a very good compromise between performance and robustness. The conclusion and some future work will be drawn in Section 5. Finally, the algebraic setting for nonlinear estimation will be presented in appendix A and applied to vehicle velocities estimation in appendix B.

\section{Control scheme}

Figure 1 graphically summarizes the whole control scheme. The stop-and-go system uses radar information and ESP accessible data to generate, via a dynamic model and a reliable leader velocity estimation, the desired acceleration. This model provides a safe and comfortable reference inter-distance between the leader and the following vehicle. A reference longitudinal acceleration is then generated as a feedforward control. Since this model is based on corrupted measures and not always valid assumptions, a feedback term is introduced. This closed-loop will not only behave as a typical PID controller, but it will also estimate linear or nonlinear unmodeled effects (road slope, wind, rolling resistance) in order to anticipate 
the controller action. The resulting control will provide an acceleration as close as possible to the desired one.

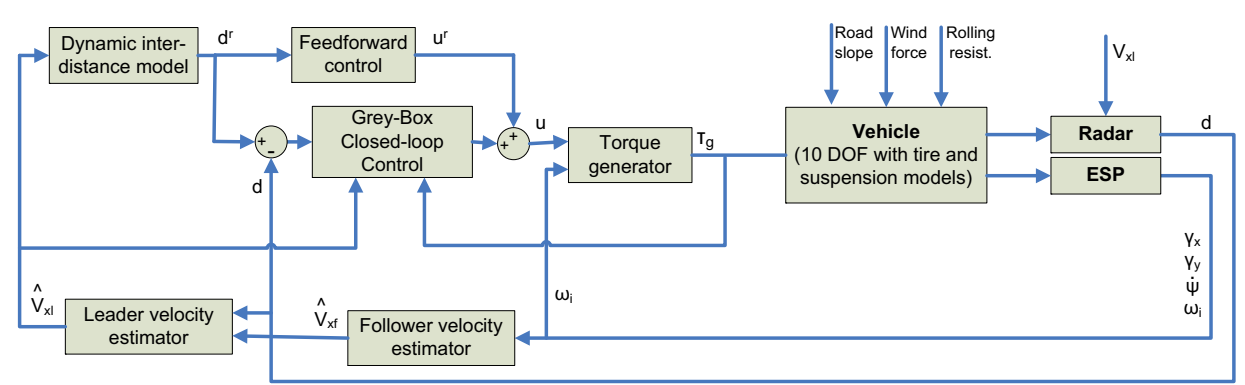

Figure 1 General Stop-and-go control scheme.

\section{Model-free control ${ }^{\mathrm{c}}$}

The difficulty for obtaining a simple but accurate mathematical model in most practical situations is well known. This explains to a large extent the great popularity of PID controllers, even if tuning them is not always an easy task (see, e.g., [Aström and Hägglund, 1995]). The proposed control strategy replaces mathematical models by local "phenomenological" differential equations, which are only valid for a short time interval, and are continuously updated.

Take a finite-dimensional SISO system

$$
E\left(t, y, \dot{y}, \ldots, y^{(\iota)}, u, \dot{u}, \ldots, u^{(\kappa)}\right)=0
$$

which is linear or not, where $E$ is a sufficiently smooth function of its arguments. Assume that for some integer $n, 0<n \leq \iota, \frac{\partial E}{\partial y^{(n)}} \not \equiv 0$. The implicit function theorem yields then locally

$$
y^{(n)}=\mathfrak{E}\left(t, y, \dot{y}, \ldots, y^{(n-1)}, y^{(n+1)}, \ldots, y^{(\iota)}, u, \dot{u}, \ldots, u^{(\kappa)}\right)
$$

This equation becomes by setting $\mathfrak{E}=F+\alpha u$ :

$$
y^{(n)}=F+\alpha u
$$

where

- $\alpha \in \mathbb{R}$ is a non-physical constant parameter, which is chosen by the engineer in such a way that $F$ and $\alpha u$ are of the same magnitude,

- $F$ is determined thanks to the knowledge of $u, \alpha$, and of the estimate of $y^{(n)}$.

${ }^{\mathrm{c}}$ See [Fliess and Join, 2008] for more details. 
Remark 3.1. A large literature exists on the estimation of derivatives for noisy signals (cf e.g. [Fliess et al, 2008] and the references therein). However, a new approach to estimate derivatives from noisy measurements, which will be detailed in appendix A.2, has been implemented in this work. It will not only permit to estimate $y^{(n)}$ for the proper computation of $F$, but it will also be used in leader velocity estimation from radar signals, or in wheel velocity derivative estimation.

Remark 3.2. A system might only be partially unknown as in Sect. 4.5. It is straightforward to adapt the previous method in this case.

In all the known examples until today, $n$ was chosen to be equal to 1 or 2 in Eq. (1). If $n=2$, the desired behavior is obtained via the intelligent PID controller, which is of the form

$$
u=\frac{1}{\alpha}\left(\ddot{y}_{*}-F+K_{P} e+K_{I} \int e d t+K_{D} \frac{d e}{d t}\right)
$$

where

- $y_{*}$ is a reference trajectory which is selected as in flatness-based control (see, e.g., [Fliess et al, 1995; Sira-Ramírez and Agrawal, 2004]),

- $e=y-y_{*}$ is the tracking error,

- $K_{P}, K_{I}, K_{D} \in \mathbb{R}$ are suitable gains, the tuning of which is quite straightforward.

\section{High-level loop control}

The feedforward high level control will be briefly recalled to point out its main features and the fundamental limitations that have been addressed in the present work:

- The closed-loop control is not at all robust to radar noisy measurements.

- The longitudinal velocity of the leader vehicle has to be estimated to introduce it as an input in the reference dynamic model. To achieve this task not only inter-distance time derivative is needed, but also a good velocity estimation of the following vehicle. In very demanding situations, this estimation is not an easy task.

- When sudden accelerations/decelerations are needed, the corresponding openloop engine/brake torques may be hard to compute.

After a brief introduction to vehicle longitudinal dynamics, the next subsections will detail how the first and third issues have been addressed, under the algebraic framework presented in Section A. However, the way the follower velocity estimation has been treated will be presented in appendix B. 


\subsection{Vehicle longitudinal dynamics}

A force balance along the vehicle longitudinal axis (cf. [Rajamani, 2005]) yields

$$
M \ddot{x}=F_{x_{f}}+F_{x_{r}}-F_{a}-R_{x_{f}}-R_{x_{r}}-m g \sin \theta
$$

where $F_{x_{f}}, F_{x_{r}}$ are respectively the front and rear longitudinal tire forces, $R_{x_{f}}$ and $R_{x_{r}}$ the front and rear tire forces dues to rolling resistance, $\theta$ the angle of inclination of the road, and $F_{a}$ is the longitudinal aerodynamic drag force.

The rolling resistance forces are often modeled as a time-varying linear function of normal forces on each tire, i.e. $R_{x}=k F_{z}$, with $\mathrm{k}$ the rolling resistance coefficient.

The aerodynamic forces can be written as

$$
F_{a}=\frac{1}{2} \rho C_{d} A_{F}\left(V_{x}+V_{w i n d}\right)^{2}
$$

with $\rho$ is the mass density of air, $C_{d}$ is the aerodynamic drag coefficient, $A_{F}$ is the frontal area of the vehicle (the projected area of the vehicle in the direction of travel) and $V_{x}, V_{\text {wind }}$ are respectively the longitudinal vehicle and wind velocities.

Finally, Pacejka model [Pacejka and Baker, 1991] is used for longitudinal tire/road interaction forces $F_{x}$. They depend on many factors, but essentially on longitudinal slip and normal forces. These normal forces will be computed as realistically as possible $^{\mathrm{d}}$ within a 10 d.o.f vehicle model.

\subsection{Feedforward control}

A reference model proposed by [Martinez and Canudas-de-Wit, 2007] will act as a feedforward term into the longitudinal high level control law. The basis of this model will be sketched in the next lines.

The inter-distance reference model describes a virtual vehicle dynamics which is positioned at a distance $d^{r}$ (the reference distance) from the leader vehicle. The reference model dynamics is given by

$$
\ddot{d}^{r}=\hat{\ddot{x}}_{l}-\ddot{x}_{f}^{r}
$$

where $\hat{\ddot{x}}_{l}$ is an estimation of the leader vehicle acceleration and

$$
\ddot{x}_{f}^{r}=u^{r}\left(d^{r}, \dot{d}^{r}\right)
$$

is a nonlinear function of the inter-distance and of its time derivative.

Introducing $\tilde{d} \triangleq d_{0}-d^{r}$ in $(5)$, where $d_{0}$ is the safe nominal inter-distance, the control problem is then to find a suitable control when $\tilde{d} \geqslant 0$ :

$$
u^{r}=u_{2}(\tilde{d}, \dot{\tilde{d}}), \tilde{d} \geqslant 0
$$

such that all the solutions of the dynamics (4) fulfill the following comfort and safety constraints:

\footnotetext{
${ }^{\mathrm{d}}$ Realistic tire forces models remain an open problem. Nevertheless, many authors have attacked it from different point of views (cf. i.e. [Pacejka and Baker, 1991] or [Canudas-de-Witt et al, 2003]).
} 
- $d^{r} \geqslant d_{c}$, with $d_{c}$ the minimal inter-distance, will guarantee collision avoidance.

- $\left\|\ddot{x}^{r}\right\| \leqslant \gamma_{\max }$, where $\gamma_{\max }$ is the maximum attainable longitudinal acceleration, depending on the driver, the vehicle and the infrastructure, will have an effect on security and comfort.

- $\left\|\dddot{x}^{r}\right\| \leqslant J_{\max }$, with $J_{\max }$ a bound on the driver desired jerk, will directly affect comfort performances.

Inspired by the theory of elasticity and mechanics of contact, [Martinez and Canudas-de-Wit, 2007] propose to use a general nonlinear damper/spring model:

$$
u_{2}=-c|\tilde{d}|^{n} \dot{\tilde{d}}-k \tilde{d}^{n}, \forall \tilde{d} \geqslant 0
$$

which, for $k=0$ (no bouncing effects) and $n=1$, can be introduced in the dynamics equation (4) to give:

$$
\ddot{\tilde{d}}=-c|\tilde{d}| \dot{\tilde{d}}-\hat{\ddot{x}}_{l}
$$

The previous equation may be analytically integrated and expressed backwards in terms of $d^{r}$ as follows, assuming that $\hat{\dot{x}}_{l}(0)=0$ :

$$
\dot{d}^{r}=\frac{c}{2}\left(d_{0}-d^{r}\right)^{2}+\hat{\dot{x}}_{l}(t)-\beta, \beta=\dot{x}_{f}^{r}(0)+\frac{c}{2}\left(d_{0}-d^{r}(0)\right)^{2} .
$$

From (5), the feedforward control law is then obtained applying

$$
\ddot{x^{r}}=u^{r}=c\left|d_{0}-d^{r}\right| \dot{d}^{r}
$$

where the inter-distance evolution comes from the numerical integration of (6).

Note that the parameters $c$ and $d_{0}$ are algebraic functions of safe and comfort parameters $d_{c}, V_{\max }, \gamma_{\max }$ (cf. [Martinez and Canudas-de-Wit, 2007]):

$$
c=\frac{27 \gamma_{\max }}{8 V_{\max }^{3}}, \quad d_{0}=\sqrt{\left(\frac{16}{27}\right)} \frac{V_{\max }^{2}}{\gamma_{\max }+d_{c}}
$$

which ensures that the constraints are fulfilled.

\subsection{Closed-loop control}

Since the physical control (brake/throttle) can neither be instantaneously nor precisely applied to track (7), and since many errors are induced by measurement noises, some kind of feedback control must be introduced

$$
u=u^{r}+H(s)\left(d^{r}-\hat{d}\right)
$$

where $H(s)$ is here a linear operator (e.g. a PID) of the measured tracking error. However, this feedback compensation leads to extremely noisy perturbed results when a derivative term is used, and to instability or important tracking errors when it is not. 
In order to avoid this kind of problem, a PD compensator ${ }^{\mathrm{e}}$ has been implemented, where inter-distance and its time-derivatives are obtained using Sect. A.2. The signal can be locally approximated by a linear polynomial $(N=1)$. Thus, $d(t)=d_{0}+d_{1} t, t \geqslant 0, d_{0}, d_{1} \in \mathbb{R}$. In the first case, an estimator for $d_{0}$ is sought; in the second one, $d_{1}$ will be estimated. If we take for instance $\nu=2$, the estimators can be written as follows :

$$
\begin{aligned}
& \hat{d}=\hat{d}_{0}=\frac{2}{T^{2}} \int_{0}^{T}(2 T-3 \tau) d(\tau) d \tau \\
& \hat{\dot{d}}=\hat{d}_{1}=\frac{-3 !}{T^{3}} \int_{0}^{T}(T-2 \tau) d(\tau) d \tau
\end{aligned}
$$

Figure 2 shows the difference between applying two discrete PD controller with a different low-pass filter and an algebraic PD controller.
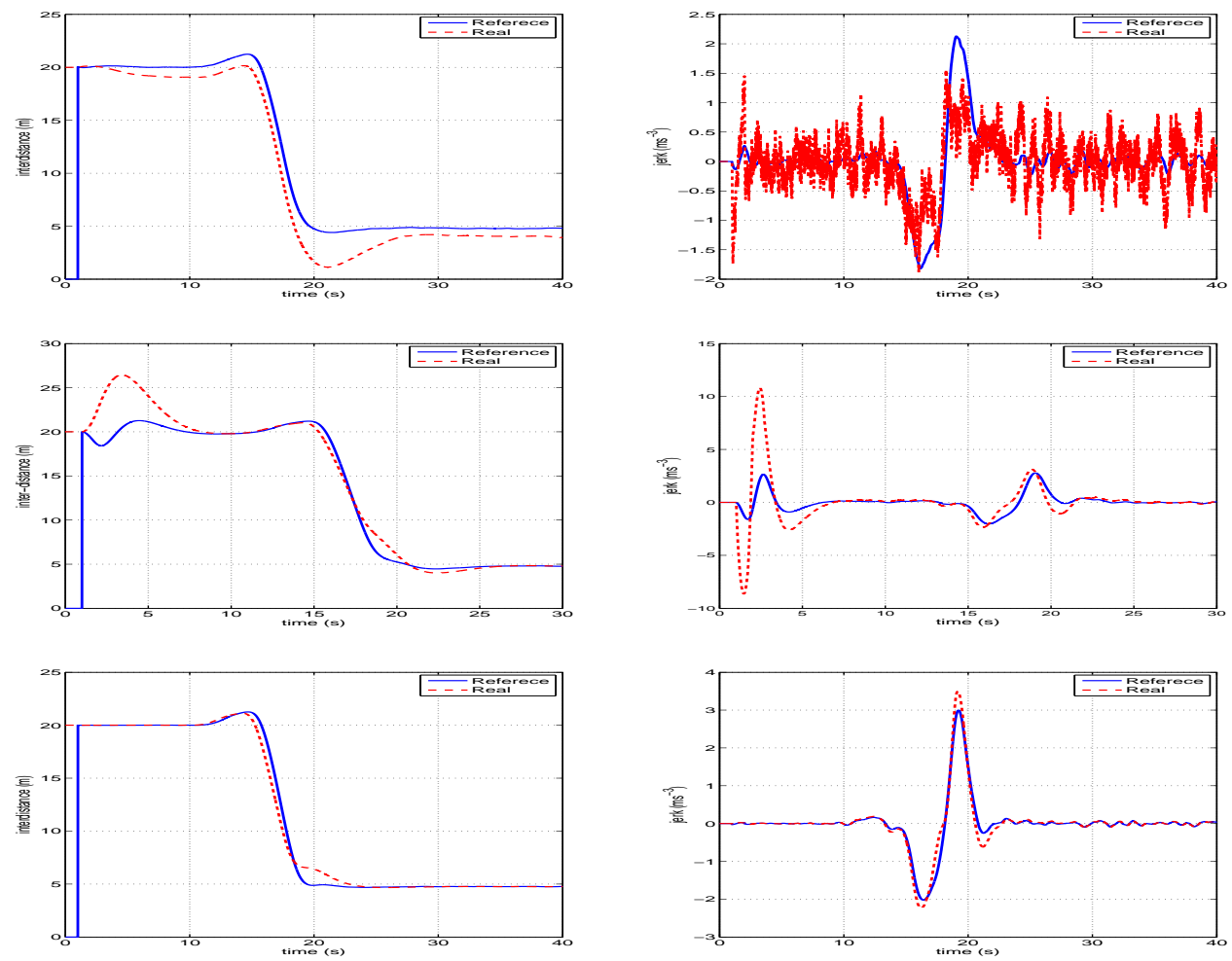

Figure 2 Inter-distance and jerks evolution with different closed-loop controllers. A discrete $\mathrm{PD}$ with low-pass filter of cut-off frequency equal to $100 \mathrm{~Hz}$ (top), another one with cut-off frequency equal to $5 \mathrm{~Hz}$ (middle) and an algebraic PD (bottom). The $P=0.7$ and $D=0.4$ parameters are identical in all cases.

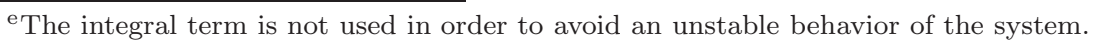




\subsection{Reference torque generation}

The wheel rotation dynamics can be written as follows

$$
I \dot{\omega}=-r F_{x}+\tau_{e}-\tau_{b}
$$

where $I$ is the rotation inertia moment, $\dot{\omega}$ the wheel angular velocity, $r$ is the tire radius, $\tau_{e}$ the applied engine torque, and $\tau_{b}$ the brake torque, both of them applied to the wheel center.

A commonly used assumption ([Hedrick et al, 1991], [Rajamani, 2005], [Nouvelière and Mammar, 2004]) consists in considering rolling without slipping, i.e. $V_{x}=R_{g} r \omega_{e}$, where $R_{g}$ is the gear ratio. However, in a stop-and-go context, where fast responses to sudden decelerations are required, this is not an acceptable hypothesis.

If a generalized wheel torque $\tau_{e b}=\tau_{e}-\tau_{b}$ is considered, it is straightforward to see its dependence on tire/road interaction forces. Therefore, a realistic estimation of this generalized torque from equation (10) turns out to be quite hard.

The sum of the 4 wheels rotation dynamics equations and of the vehicle dynamic longitudinal equation $M \gamma_{x}=\sum_{i=1}^{4} F_{x_{i}}$ yields, when $F_{a}, R_{x_{i}}$ and $\theta$ from (3) are neglected,

$$
\tau_{g}=4 \tau_{e b}=I \sum_{i=1}^{4} \dot{w}_{i}+r M \gamma_{x} .
$$

The main inconvenient for such an estimator is that a good numerical differentiator for $\dot{\omega}_{i}$ is needed. An equivalent algebraic estimator to (9) will then be used to compute $\hat{\dot{w}}_{i}$ :

$$
\hat{\dot{\omega}}=\frac{-3 !}{T^{3}} \int_{0}^{T}(T-2 \tau) \omega(\tau) d \tau .
$$

Figure 3 shows a comparison between algebraic derivatives and discrete derivatives with a low-pass filter. The processed signal is here $\omega$ because of its implication in torque generation. The results seem to be quite similar, but noise filtering is more effective with algebraic derivatives estimators.

Finally, figure 4 compares inter-distances between open-loop generated torque under no slipping assumption and open-loop torque with our dynamic estimation approach. A remarkable improvement can be obtained when this new strategy is used in demanding situations. Indeed, results shown in figure 4 are obtained with longitudinal accelerations up to $5.5 \mathrm{~ms}^{-2}$, which are rarely found in an ACC context.

\subsection{Grey-box feedback control}

The procedure described in Sect. 3 can here be applied in a particular way. Since some specific dynamics are very well known, it is worth to integrate them in our predictive scheme. Thus, the design parameter $\alpha$ become here well-known quantities. Recall the local input-output model introduced in Eq. (1) and compare 


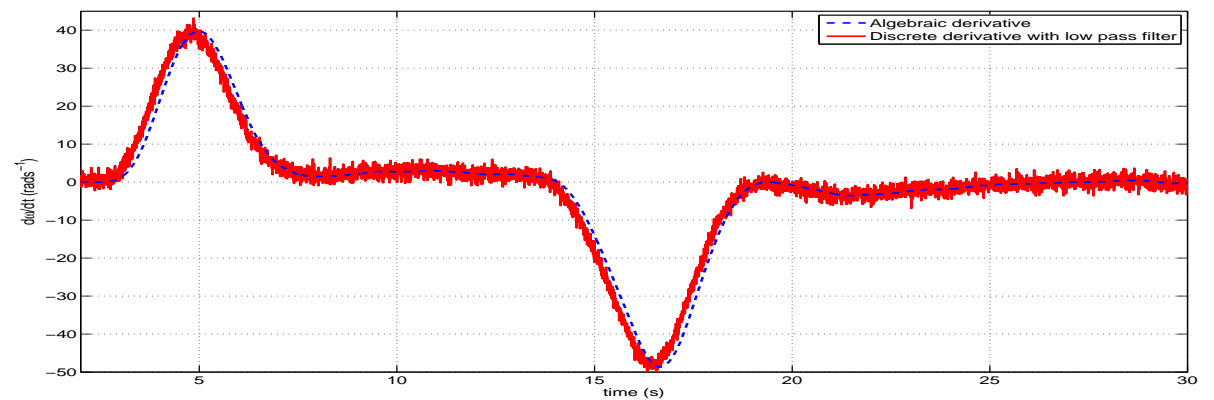

Figure 3 Wheel speed derivative computed with (a) discrete derivatives and low-pass filter, and (b) with algebraic derivative estimator.

it with the reordered torque expression (11)

$\gamma_{x}=\frac{1}{M r}\left(\tau_{g}-I \sum_{i=1}^{4} \dot{\omega}_{i}+G(t)\right), G(t)=r\left(F_{a}(t)-R_{x_{f}}(t)-R_{x_{r}}(t)-M g \sin \theta(t)\right)$

In a stop-and-go scenario, rolling without turning is considered, so that $\dot{V}_{x}=\gamma_{x}$ is verified. Therefore, the next equation can be written

$$
\dot{V}_{x}=F(t)+\alpha u(t)+\beta(t)
$$

where $F(t)=\frac{G(t)}{M r}, \alpha=\frac{1}{M r}$ and $\beta(t)=-\frac{I \sum_{i=1}^{4} \dot{\omega}_{i}(t)}{M r}$ and $u(t)=\tau_{g}(t)$ is the control variable.

The goal is to obtain an accurate closed-loop estimation of $F$. Following the theoretical ideas described in Sect. A, the procedure consists, first of all, in rewriting (12) in the operational domain, with the assumption $F=F_{0}$ in a short estimation time window,

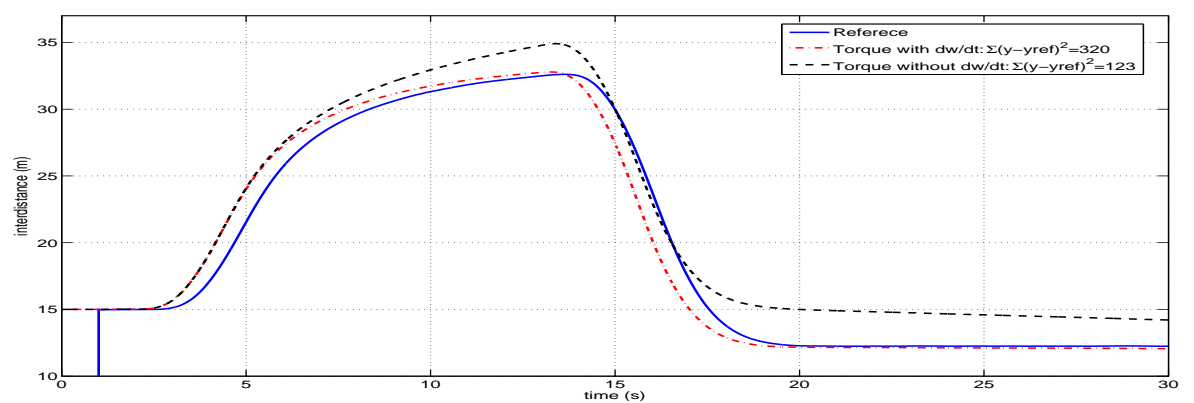

Figure 4 Comparison between torque generation under no slipping assumption, and with expression 11 . 


$$
s V_{x}-V_{0}=\frac{F_{0}}{s}+\alpha \tau_{g}(s)+\beta(s)
$$

and then applying the operator $\frac{d}{d s}$ with the aim of eliminating the initial condition $V_{0}$

$$
V_{x}+s \frac{d V_{x}}{d s}=-\frac{F_{0}}{s^{2}}+\alpha \frac{d \tau_{g}}{d s}+\frac{d \beta}{d s}
$$

Finally, $s^{-\nu}$, with $\nu=2$ is applied in order to eliminate any non causal term

$$
\frac{F_{0}}{s^{4}}=-\frac{1}{s^{2}} V_{x}-\frac{1}{s} \frac{d V_{x}}{d s}+\alpha \frac{1}{s^{2}} \frac{d \tau_{g}}{d s}+\frac{1}{s^{2}} \frac{d \beta}{d s}
$$

which, expressed backwards in the time domain, yields

$$
\hat{F}=F_{0}=\frac{3 !}{T^{3}} \int_{0}^{T}\left((-T+2 t) V_{x}(t)-(T-t) t\left(\alpha \tau_{g}(t)+\beta(t)\right)\right) d t
$$

The final closed-loop control is then, applying (2) to our case and considering rolling without turning (i.e. $V_{x}^{r}=\ddot{x}=u^{r}$ ):

$$
\tau_{g}=M r\left(u^{r}-F_{0}+\frac{I}{M r} \sum_{i=1}^{4} \dot{\omega}_{i}+K_{P} e+K_{D} \dot{e}\right), e=d-d^{r}
$$

When the complete strategy is tested on a quite demanding scenario ${ }^{\mathrm{f}}$, the interdistance model reference is pretty well tracked (see figure 5), the follower acceleration remains under the comfort constraints, and consequently, the jerk bounds are also guaranteed (cf. [Martinez and Canudas-de-Wit, 2007]). Furthermore, the generalized torque applied to the vehicle seems very robust to noise perturbation.

However, the most important source of uncertainty comes from road conditions. Thus, if rolling resistance, aerodynamic efforts and a sloped road are introduced, the results are slightly different. Figure 6 a shows that even if the inter-distance trends are already very well respected, a variable bias cannot be annihilated with the "standard" control. The grey-box control strategy has been applied in order to obtain more robust results. The dashed line in figure 6 a represents the tracking performance when the estimator $F$ of global disturbances is introduced. A considerable improvement (almost 400\%) is obtained when the global effects of disturbances are estimated via equation (15).

It can be appreciated from figure $6 \mathrm{~b}$ that road slope, rolling resistance and aerodynamic forces are pretty well estimated in an overall term $\hat{F}=F_{0}$. Note that aerodynamic forces are not very significant when compared with the road slope. However, big wind gusts can appear at high speeds. In this case, a reliable and fast estimator should applied. Figure 7 shows the behavior of control law proposed in (16) when severe wind gusts longitudinally knock the car. It can be appreciated that our control is much more robust when $F$ is estimated. Moreover, the time window estimation size $T_{e}$ can be used as a tuning parameter for safety or comfort.

\footnotetext{
${ }^{\mathrm{f}}$ Several heavy accelerations/decelerations are applied to the vehicle on a flat road, where neither rolling resistance nor aerodynamic forces are considered.
} 

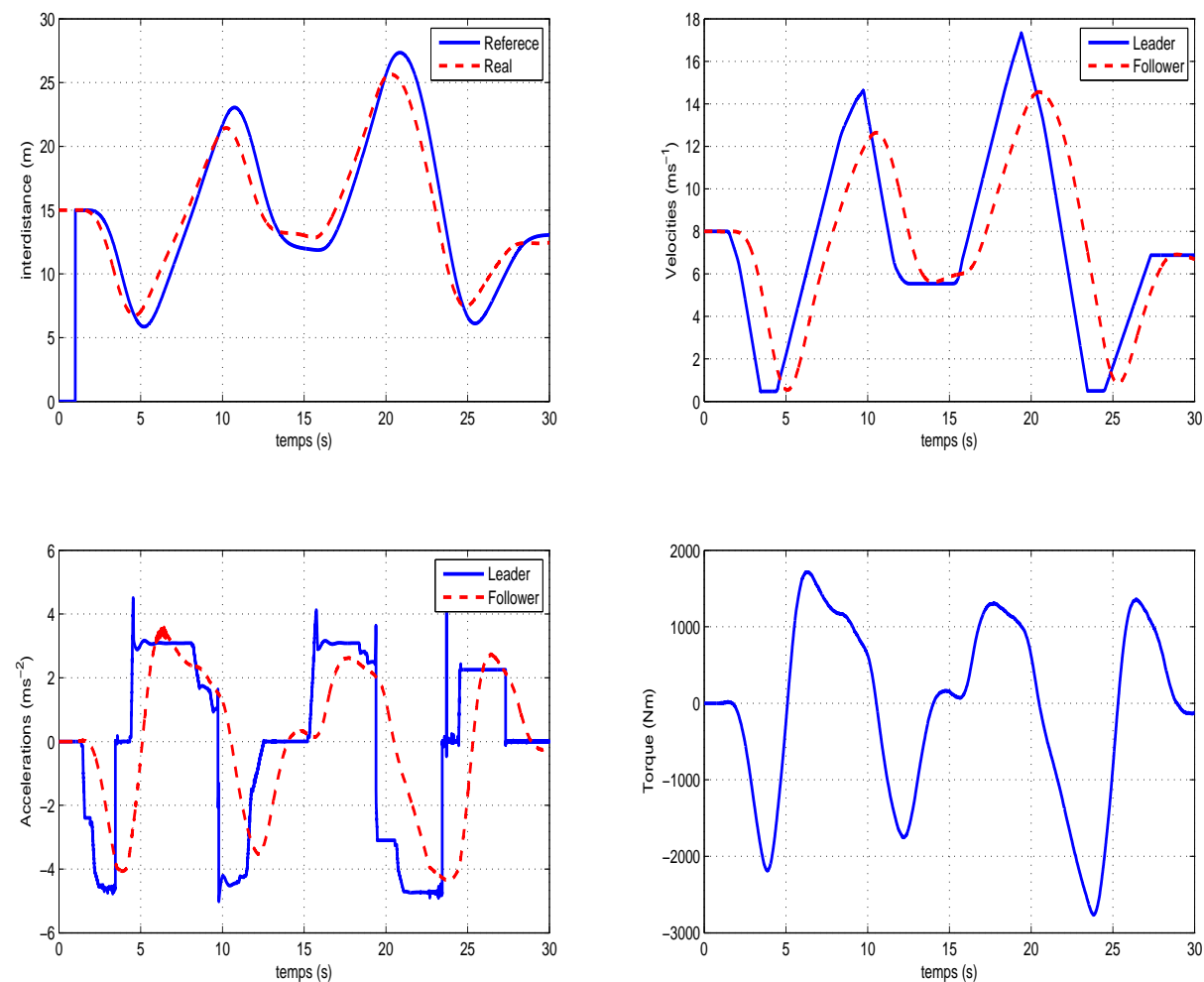

Figure 5 Inter-distance, velocity, acceleration and generalized torque for highly demanding scenario (up to $5 \mathrm{~ms}^{-1}$ ).

When tracking performance is more important than comfort (sudden changes in acceleration), a small window will be used. If important jerks are not desired, a bigger window estimation will be more appropriate.

\section{Concluding remarks}

It can be seen that, as expected, our method leads to a closed-loop behavior which is robust with respect to noises and unmodeled dynamics. To be complete, we have now to deal with the low-level part describing the engine and brake dynamics to generate the physical control variables: the throttle angle and brake pressure (see already [Choi et al, 2009]). This work is under study.

Moreover, to take into account the road friction conditions, a reliable estimation of the friction coefficient is necessary. This problem is also under investigation (see [Villagra et al, 2009]). 

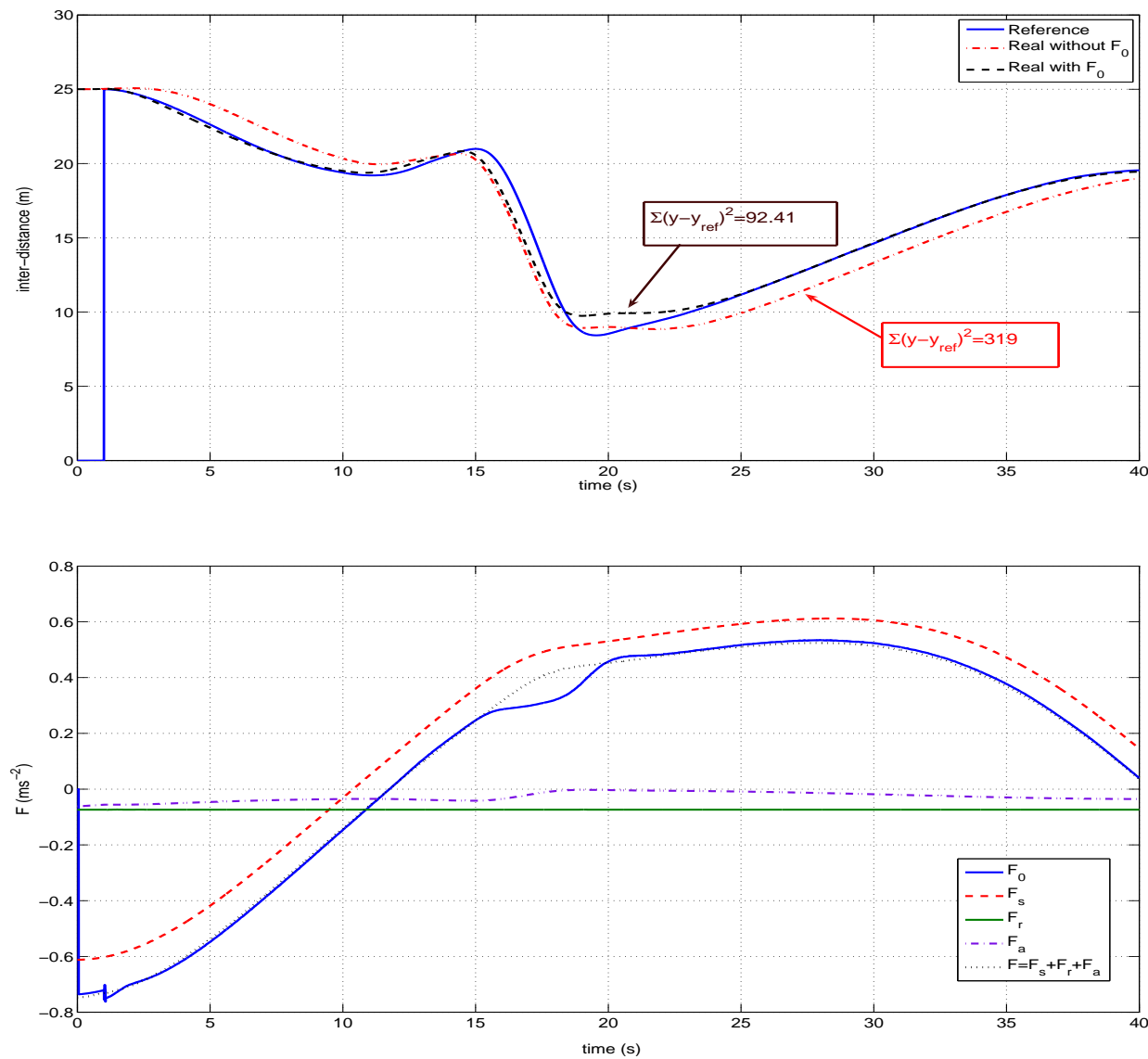

Figure 6 (a) Inter-distance evolution with and without $F$ estimation. Comparison between real $F$ and its estimate $F_{0}$. (b) Aerodynamic $\left(F_{a}\right)$, road slope $\left(F_{s}\right)$ and rolling resistance $\left(F_{r}\right)$ terms are also depicted.

\section{References}

K.J. Aström, T. Hägglund, Advanced PID Controllers, Instrument Soc. Amer., 2006.

M. Brackstone and M. McDonald, 'Car-following: A historical review', Transportation Research F, Vol. 2, pp. 181-196, 2000.

C. Canudas-de-Witt, P. Tsiotras, E. Velenis, M. Basset and G. Gissinger, 'Dynamic friction models for road/tire longitudinal interaction', Vehicle System Dynamics, Vol. 39, pp. 189-226, 2003.

C. Chien and P. Ioannou, 'Automatic vehicle-following' Proc. Amer. Contr. Conf. pp. 1748-1752, 1992. 

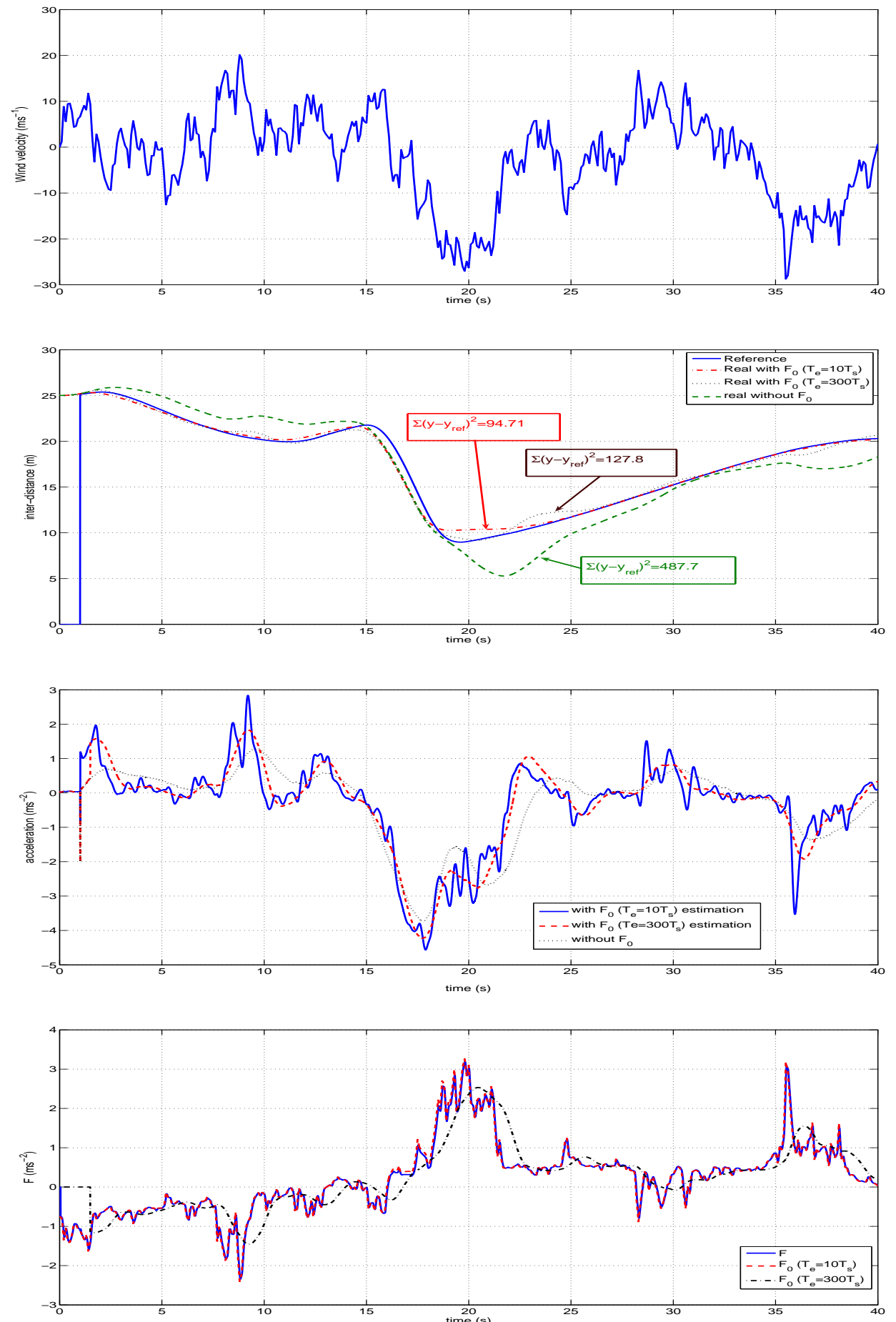

Figure 7 (a) Wind velocity. (b) Inter-distance evolution with different sliding windows for $F$ estimation. (c) Longitudinal acceleration with different estimation time windows. (d) F estimation 
S. Cho and J.K. Hedrick, 'Vehicle Longitudinal Control Using an Adaptive observer for automated highway systems', Proc. American Control Conf., pp. 3106-3110, Seattle, 1995.

S.Choi, B. d'Andréa-Novel, M. Fliess, H. Mounier and J. Villagra, 'Greybox feedback control of automotive engine and brake for stop-and-go scenario', European Control Conference, Budapest, Hungary, 2009 (available at http://hal.inria.fr/inria-00395393/en/).

S. Diop, and M. Fliess, 'On nonlinear observability', Proc. $1^{\text {st }}$ Europ. Control Conf., pp. 152-157, Grenoble, 1991.

S. Diop, and M. Fliess, Nonlinear observability, identifiability and persistent trajectories', Proc. 36 ${ }^{\text {th }}$ IEEE Conf. Decision Control, pp. 714-719, Brighton, 1991.

M. Fliess, 'Analyse non standard du bruit', C.R Acad. Sc. Paris, ser. I, vol. 342, pp. 797-802, 2006.

M. Fliess, C. Join, 'Commande sans modèle et commande à modèle restreint', e-STA, vol. 5, 2008, pp 1-23 (available at http://hal.inria.fr/inria-00288107/en/).

M. Fliess, C. Join, and H. Sira-Ramírez, 'Non-linear estimation is easy', Internat J. Modelling Identification Control, Vol. 4, pp. 12-27, 2008.

M. Fliess, J. Lévine, P. Martin and P. Rouchon, Flatness and defect of non-linear systems: introductory theory and examples, Int. J. Control, Vol. 61, pp. 13271361, 1995.

Fliess and H. Sira-Ramírez, 'An algebraic framework for linear identification', ESAIM Control Optim. Calc. Variat., Vol. 9, pp. 151-168, 2003.

M. Fliess and H. Sira-Ramírez, 'Closed-loop parametric identification for continuous-time linear systems via new algebraic techniques', in $\mathrm{H}$. Garnier \& L. Wang (Eds.): Identification of Continuous-time Models from Sampled Data, Springer, pp. 362-391, 2008 (available at http://hal.inria.fr/inria-00114958/en/).

J.C. Gerdes and J.K. Hedriek, 'Vehicle speed and spacing control via coordinated throttle and brake actuation', Control Eng. Practice, Vol. 5 No. 11, pp. 1607-1614, 1997.

S. Germann and R. Isermann, 'Nonlinear distance and cruise control for passenger cars', Proc. Amer. Control Conf., pp. 3081-3085, Seattle, 1995.

J. K. Hedrick, D. McMahon, V. Narendran, D. Swaroop, 'Longitudinal Vehicle Controller Design for IVHS Systems', Proc. Amer. Control Conf., pp. 3107-3112, Boston, 1991.

T. Hiraoka, T. Kunimatsu, O. Nishihara and H. Kumamoto, 'Modeling of driver following behavior based on minimum-jerk theory', Proc. $12^{\text {th }}$ World Congress ITS, paper 3416, San Francisco, 2005. 
P. Ioannou and Z. Xu, 'Throttle and Brake Control Systems for Automatic Vehicle Following' IVHS Journal, Vol.1 No. 4, pp.345-377, 1994.

M. Liubakka, D. Rhode, J. Winkelman, and P. Kokotovic, 'Adaptive automotive speed control', IEEE Trans. Automat. Control, Vol. 38, pp. 146156, 1993.

J. Martinez and C. Canudas-de-Wit, 'A Safe Longitudinal Control for Adaptive Cruise Control and Stop-and-Go Scenarios', IEEE Trans. Control Systems Technology, Vol. 15, pp. 246-258, 2007.

M. Mboup, C. Join and M. Fliess, 'Numerical differentiation with annihilators in noisy environment', Numer. Algorithm., Vol. 4, pp 439-467, 2009.

J. E. Naranjo, C. González, R. García and T. de Pedro, 'ACC+Stop\&Go Maneuvers With Throttle and Brake Fuzzy Control', IEEE Trans. Intelligent Transportation Systems, Vol. 7, No. 2, pp 213-225, 2006.

L. Nouvelière and S. Mammar, 'Experimental vehicle longitudinal control using a second order sliding mode technique', Control Eng. Practice, Vol. 15, pp. 943-953, 2007.

K. Oda, H. Takeuchi, M. Tsujii and M. Ohba, 'Practical estimator for self-tuning automotive cruise control' Proc. American Control Conf., Boston, pp. 2066-2071, 1991.

H. Pacejka, E. Baker, 'The magic formula tyre model', Proc. $1^{\text {st }}$ Internat. Coll. Tyre Models Vehicle System Analysis, pp. 1-18, 1991.

M. Persson, F. Botling, E. Hesslow and R. Johansson, 'Stop \& Go Controller for Adaptive Cruise Control', Proc. of the IEEE International Conference on Control Applications, pp 1692-1697, Hawaii, 1999.

R. Rajamani, Vehicle Dynamics And Control, Springer, 2005.

H. Raza, Z. Xu, B. Yang, and P. Ioannou, 'Modeling and control design for a computer-controlled brake system', IEEE Trans. Control Syst. Technol., Vol. 5, pp. 279-296, 1997.

H. Sira-Ramírez, and S.K. Agrawal, Differentially Flat Systems, Marcel Dekker, 2004.

D. Swaroop, K. Hedrick, C. Chien, and P. Ioannou, 'Comparison of spacing and headway control laws for automatically controlled vehicles', Vehicle Syst. Dyn., Vol. 23, pp. 597625, 1994.

A. Vahidi and A. Eskandarian, 'Research Advances in Intelligent Collision Avoidance and Adaptive Cruise Control', IEEE Trans. Intelligent Transportation Systems, Vol. 4, pp 143-153, 2003.

J. Villagra, B. d'Andréa-Novel, M. Fliess and H. Mounier, 'Estimation of longitudinal and lateral vehicle velocities: an algebraic approach', American Control Conference, pp. 3941-3946, Seattle, 2008. 
J. Villagra, B. d'Andréa-Novel, M. Fliess and H. Mounier, 'A diagnosis-based approach for tire forces and friction estimation', submitted to Control Engineering Practice.

K. Yi and J. Chung, 'Nonlinear Brake Control for Vehicle CW/CA Systems', IEEE/ASME Transactions on Mechatronics, Vol. 6, No. 1, pp. 17-25, 2001.

K. Yi and I. Moon, 'A Driver-Adaptive Stop-and-Go Cruise Control Strategy', Proc. of IEEE Int. Conf. on Networking. Sensing $\&$ Control, pp. 601-601, Taipei, 2004.

K. Yosida, Operational Calculus: A Theory of Hyperfunctions, Springer, New York, 1984 (translated from the Japanese).

K. Youcef-Toumi, Y. Sasage, Y. Ardini, and S. Huang, 'Application of time delay control to an intelligent cruise control system' Proc. American Control Conf., pp. 1743-1747, Chicago, 1992.

\section{A Algebraic setting for parametric identification, nonlinear estimation and model-free control}

\section{A.1 Linear identification ${ }^{\mathrm{g}}$}

Let us first introduce some usual notations from operational calculus (see, e.g., [Yosida, 1984]):

- $s$ is the differentiation operator,

- $\frac{d}{d s}$ corresponds in the time domain to the multiplication by $-t$.

Write

- $\mathbb{R}(s)$ the field of rational functions in the variable $s$ with real coefficients,

- $\mathbb{R}(s)\left[\frac{d}{d s}\right]$ is the set of linear differential operators of the form

$$
\sum_{\text {finite }} a_{\alpha} \frac{d^{\alpha}}{d s^{\alpha}} \quad a_{\alpha} \in \mathbb{R}(s)
$$

This is a noncommutative ring since $\frac{d}{d s} s-s \frac{d}{d s}=\left[\frac{d}{d s} s, s \frac{d}{d s}\right]=1$.

A finite set $\Theta=\left\{\theta_{1}, \ldots, \theta_{r}\right\}$ of constant parameters is said to be linearly identifiable with respect to a finite set $X=\left\{x_{1}, \ldots, x_{k}\right\}$ of signals, the input and output variables of a linear system for instance, if, and only if, it reads in the operational domain

$$
\mathcal{P}\left(\begin{array}{c}
\theta_{1} \\
\vdots \\
\theta_{r}
\end{array}\right)=\mathcal{Q}
$$

where

\footnotetext{
${ }^{\mathrm{g}}$ See [Fliess and Sira-Ramírez, 2003, 2008] for more details.
} 
- $\mathcal{P}$ and $\mathcal{Q}$ are respectively $r \times r$ and $r \times 1$ matrices,

- the entries of $\mathcal{P}$ and $\mathcal{Q}$ belong to $\operatorname{span}_{\mathbb{R}(s)\left[\frac{d}{d s}\right]}\left(1, x_{1}, \ldots, x_{k}\right)$,

- $\operatorname{det} \mathcal{P} \neq 0$.

Replace in Eq. (17) $x_{i}, i=1, \ldots, k$, by $y_{i}=x_{i}+\varpi_{i}$, where $\varpi_{i}$ is some additive perturbation. Eq. (17) reads

$$
\mathcal{P}\left(\begin{array}{c}
\theta_{1} \\
\vdots \\
\theta_{r}
\end{array}\right)=\mathcal{Q}+\mathcal{Q}^{\prime}
$$

where $\mathcal{Q}^{\prime}$ is a $r \times 1$ matrix with entries depending now on $\varpi_{i}, i=1, \ldots, k$.

A perturbation $\varpi$ is said to be structured if, and only if, there exists $\pi \in \mathbb{R}(s)\left[\frac{d}{d s}\right]$, $\pi \neq 0$, such that $\pi \varpi=0$. It means in other words that $\varpi$ satisfies a linear differential equation with polynomial coefficients. If $\varpi_{1}, \ldots, \varpi_{k}$ are structured, it can be shown that there exists $\Delta \in \mathbb{R}(s)\left[\frac{d}{d s}\right], \Delta \neq 0$, such that by multiplying both sides of Eq. (18) by $\Delta$ annihilates the structured perturbations:

$$
\Delta \mathcal{P}\left(\begin{array}{c}
\theta_{1} \\
\vdots \\
\theta_{r}
\end{array}\right)=\Delta \mathcal{Q}
$$

Multiplying both sides of Eq. (19) by suitable proper rational functions in $\mathbb{R}(s)$ yields proper rational functions in all the coefficients.

The unstructured perturbations are viewed as highly fluctuating noises, which are attenuated by low-pass filters, such as $s^{-\nu}$, where $\nu \geq 0$ is large enough (see [Fliess, 2006] for more details).

\section{A.2 Nonlinear estimation and numerical differentiation ${ }^{\mathrm{h}}$}

According to the definition given in [Diop and Fliess, 1991a,b], a nonlinear inputoutput system is observable if, and only if, any system variable, a state variable for instance, is a differential function of the control and output variables, i.e., a function of those variables and their derivatives up to some finite order. This definition is easily generalized to parametric identifiability: a parameter is identifiable if, and only if, it is a differential function of the control and output variable. Following [Fliess et al, 2008] we will say more generally that an unknown quantity may be determined if, and only if, it is expressible as a differential function of the control and output variables. Nonlinear estimation and identification boils down then to numerical differentiation, i.e., to the obtention of fast and robust derivatives estimations of noisy time signals.

Start with a polynomial time function $x_{N}(t)=\sum_{\nu=0}^{N} x^{(\nu)}(0) \frac{t^{\nu}}{\nu !} \in \mathbb{R}[t], t \geq 0$, of degree $N$. The usual notations of operational calculus (see, e.g., [Yosida, 1984]) yield

$$
X_{N}(s)=\sum_{\nu=0}^{N} \frac{x^{(\nu)}(0)}{s^{\nu+1}}
$$

\footnotetext{
${ }^{\mathrm{h}}$ See [Fliess et al, 2008; Mboup et al, 2009] for more details.
} 
Multiply both sides by positive powers of $\frac{d}{d s}$. The quantities $x^{(\nu)}(0), \nu=0,1, \ldots, N$, which are linearly identifiable according to Sect. A.1, satisfy the following triangular system of linear equations:

$$
\frac{d^{\alpha} s^{N+1} X_{N}}{d s^{\alpha}}=\frac{d^{\alpha}}{d s^{\alpha}}\left(\sum_{\nu=0}^{N} x^{(\nu)}(0) s^{N-\nu}\right) \quad 0 \leq \alpha \leq N-1
$$

Multiplying both sides of Eq. (20) by $s^{-\bar{N}}, \bar{N}>N$, permit to get rid of time derivatives, i.e., of $s^{\mu} \frac{d^{\iota} X_{N}}{d s^{L}}, \mu=1, \ldots, N, 0 \leq \iota \leq N$.

Consider now an analytic time function, defined by the power series $x(t)=$ $\sum_{\nu=0}^{\infty} x^{(\nu)}(0) \frac{t^{\nu}}{\nu !}$, which is assumed to be convergent around $t=0$. Approximate $x(t)$ by the truncated Taylor expansion $x_{N}(t)=\sum_{\nu=0}^{N} x^{(\nu)}(0) \frac{t^{\nu}}{\nu !}$ of order $N$. Good estimates of the derivatives are obtained by the same calculations as above.

Remark A.1. A most elegant and powerful algorithmic procedure for obtaining a corresponding numerical differentiator is provided in [Mboup et al, 2009]. It will be exploited in the sequel.

\section{B Velocity estimation}

The proposed strategy ${ }^{\mathrm{i}}$ is based on the exploitation of accessible data (lateral and longitudinal accelerations $\gamma_{y}, \gamma_{x}$ and yaw rate $\dot{\psi}$ ) at their best, and equations

$$
\left\{\begin{array}{l}
\gamma_{x}(t)=\dot{V}_{x}(t)-\dot{\psi}(t) V_{y}(t) \\
\gamma_{y}(t)=\dot{V}_{y}(t)+\dot{\psi}(t) V_{x}(t)
\end{array}\right.
$$

by means of diagnosis tools (see for instance [Fliess et al, 2008]). Let us consider velocities $\left(V_{x}, V_{y}\right)$ as the sum of an ideal term $\left(R_{x}, R_{y}\right)$ and a "disturbing" one $\left(G_{x}, G_{y}\right)$

$$
\left\{\begin{array}{l}
V_{x}(t)=R_{x}(t)+G_{x}(t) \\
V_{y}(t)=R_{y}(t)+G_{y}(t)
\end{array}\right.
$$

where :

- $R_{x}=r \omega_{t}$, with $r$ the static wheel radius and $\omega_{t}=\frac{1}{4} \sum_{i=1}^{4} \omega_{i}$, the mean of the four wheel's rotation speed;

- $R_{y}=-L_{1} \dot{\psi}$, with $L_{1}$ the vehicle front wheelbase.

Remark B.1. The difference between faultless and faulty behavior appears in a quite natural way in longitudinal dynamics. Concerning lateral dynamics, the sideslip angle expression on the front axle's center $\beta_{1}$ can be used to obtain an equation similar to (22) :

$$
\beta_{1}=\arctan \left(\frac{V_{y}+L_{1} \dot{\psi}}{V_{x}}\right) \Rightarrow V_{y}=-L_{1} \dot{\psi}+V_{x} \tan \left(\beta_{1}\right)
$$

\footnotetext{
${ }^{i}$ See [Villagra et al, 2008] for all details.
} 
Differentiating equation (22) and replacing $\dot{V}_{x}$ and $\dot{V}_{y}$ by their corresponding expressions in (21) yields :

$$
\begin{aligned}
& \dot{R}_{x}=\dot{\psi} R_{y}-\dot{G}_{x}+\dot{\psi} G_{y}+\gamma_{x} \\
& \dot{R}_{y}=-\dot{\psi} R_{x}-\dot{G}_{y}-\dot{\psi} G_{x}+\gamma_{y}
\end{aligned}
$$

so that the following differential system on $G_{x}$ and $G_{y}$ can be written :

$$
\begin{aligned}
\dot{G}_{x}(t) & =\dot{\psi}(t) G_{y}(t)-L_{1} \dot{\psi}^{2}(t)-r \dot{\omega}_{t}(t)+\gamma_{x}(t) \\
\dot{G}_{y}(t) & =-\dot{\psi}(t) G_{x}(t)-\dot{\psi}(t) r \omega_{t}(t)+L_{1} \ddot{\psi}(t)+\gamma_{y}(t) \\
G_{x}\left(t_{0}\right) & =0, G_{y}\left(t_{0}\right)=0
\end{aligned}
$$

Let us now use equation (21) and information provided by the integration of system (23) to obtain $V_{x}$ and $V_{y}$ estimations with the following algorithms 1 and 2.
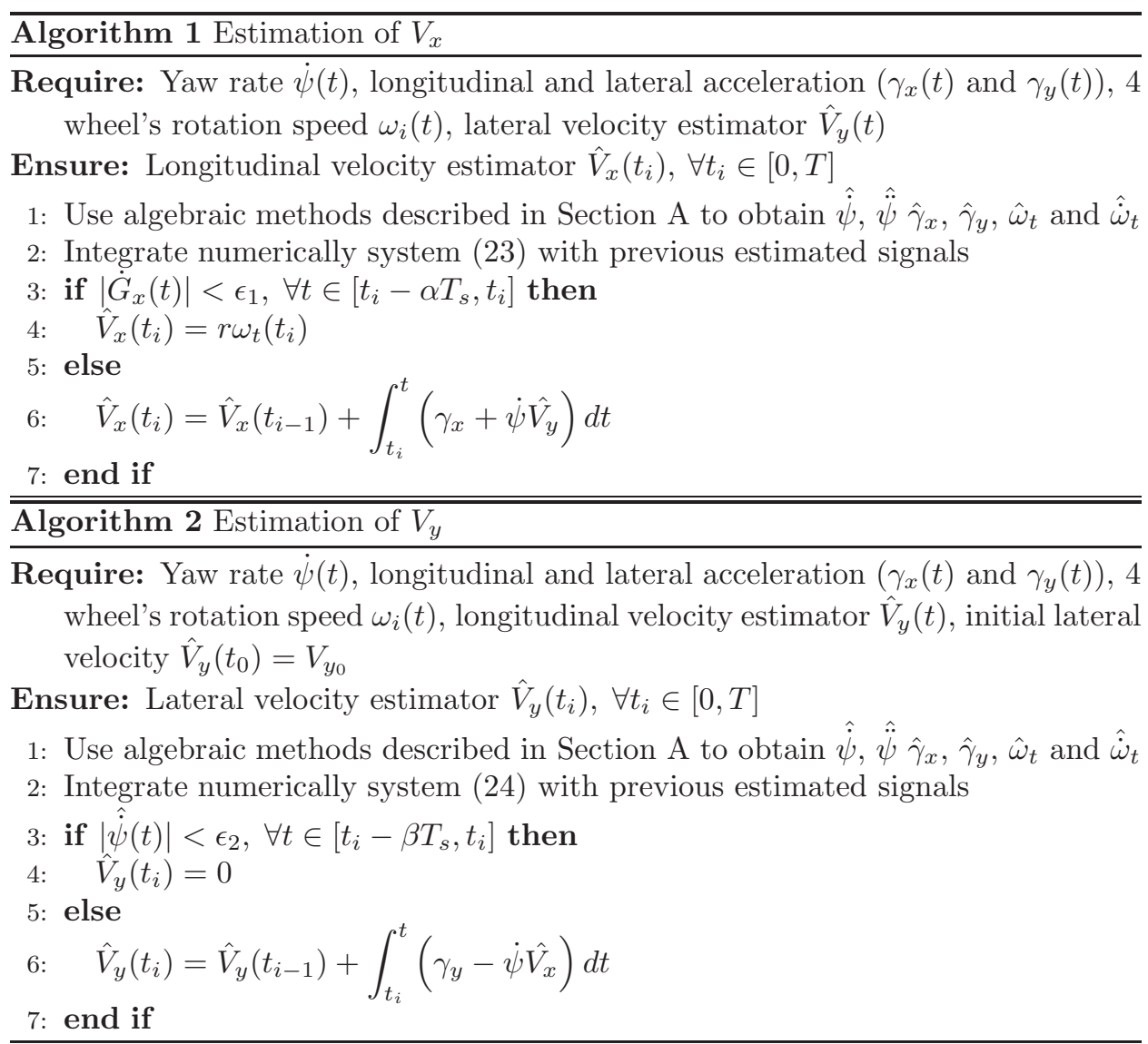

Figure 8 shows the simulation results on a relatively normal road, where the friction coefficient is $\mu=0.7$. The estimators perform pretty well in a general way. Figure 9 show the estimator's behavior on a situation where the driver action is identical, but the friction coefficient is lower $(\mu=0.5)$. 
Remark that in both cases longitudinal velocity is rather well estimated and lateral velocity respects the trends previously shown. Nevertheless, lateral velocity is much more sensitive to a good initialization and to long integration periods when it differs significantly from zero. Note that, as a consequence of this, estimation errors on $V_{x}$ have a larger influence on $V_{y}$ estimation than conversely.
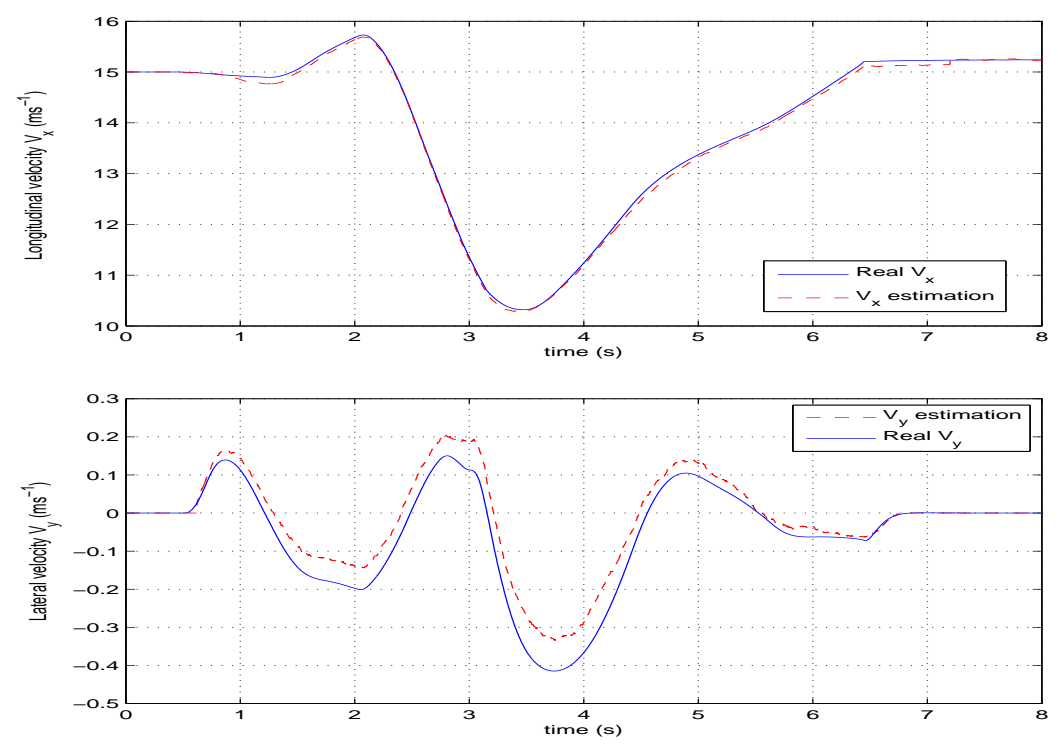

Figure 8 Real and estimated longitudinal (top) and lateral (bottom) velocities with a friction coefficient of $\mu=0.7$
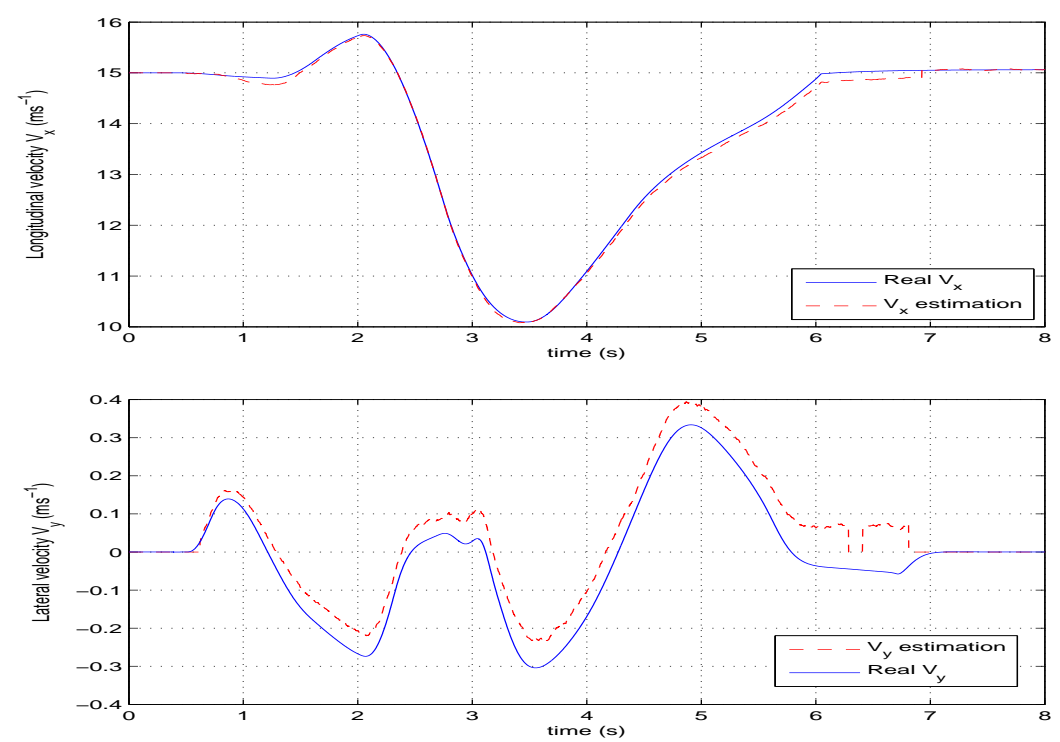

Figure 9 Real and estimated longitudinal (top) and lateral (bottom) velocities with a friction coefficient of $\mu=0.5$ 